\title{
Puerarin promotes the viability and differentiation of MC3T3-E1 cells by miR-204-regulated Runx2 upregulation
}

\author{
XIU-QIN ZHAN $^{1}$, XIANG-WEI ZENG ${ }^{1}$, YING-YING ZHANG ${ }^{1}$, QIAN FENG $^{1}$, \\ FENG-MING ZHAO ${ }^{1}$, ZE-QUN JIANG ${ }^{1}$ and CHAO SUN ${ }^{2}$ \\ ${ }^{1}$ School of Medicine and Life Science; ${ }^{2}$ The First Clinical Medical School, Nanjing \\ University of Chinese Medicine, Nanjing, Jiangsu 210023, P.R. China
}

Received August 26, 2016; Accepted May 15, 2017

DOI: $10.3892 / \mathrm{mmr} .2017 .7386$

\begin{abstract}
Puerarin has attracted increasing attention because of its beneficial effects on anti-osteoporosis, but the molecular mechanisms underlying its actions on osteoblasts are not fully understood. The current study aimed to investigate the effect of puerarin on the cell viability and differentiation of mouse MC3T3-E1 osteoblast-like cells in vitro and its underlying mechanisms. The results indicated that $0.01,0.1$ and $1 \mathrm{mg} / \mathrm{ml}$ puerarin significantly promoted the viability of osteoblasts, enhanced alkaline phosphatase (ALP) activity and increased the expression of transforming growth factor- $\beta 1, \operatorname{Smad} 2$, Smad3 and Runt-related transcription factor (Runx)2. Micro (mi)RNA target prediction programs predicted that miR-204 may directly target Runx2. Following treatment with $0.1 \mathrm{mg} / \mathrm{ml}$ puerarin for $48 \mathrm{~h}$, the expression level of miR-204 was downregulated. Besides, miR-204 dramatically repressed the luciferase activity of wildtype Runx2 3'-UTR transfected cells, but not that of the mutant ones. Overexpression of miR-204 in osteoblasts significantly decreased the protein expression of Runx2, while inhibition of miR-204 enhanced Runx2's expression. In addition, overexpression of miR-204 inhibited the cell viability and ALP activity of osteoblasts, while inhibition of miR-204 had the opposite effect. The results suggested that puerarin may promote MC3T3-E1 osteoblast-like cell viability and differentiation, which may be related to the downregulation of miR-204 and the following activation of Runx2.
\end{abstract}

\section{Introduction}

Osteoporosis is a chronic metabolic disease, defined as a skeletal disorder characterized by low bone mass density and

Correspondence to: Professor Xiu-Qin Zhan, School of Medicine and Life Science, Nanjing University of Chinese Medicine, 138 Xianlin Avenue, Nanjing Xianlin University City, Nanjing, Jiangsu 210023, P.R. China

E-mail: xqzhan14@sina.com

Key words: MC3TC-E1 cells, microRNA-204, viability, differentiation, puerarin, Runx2 microarchitectural deterioration, which predisposes patients to fracture (1). It is generally believed that osteoporosis is the failure of dynamic balance between the osteoblastic bone formation and osteoclastic bone resorption, and the functional activities of osteoblasts serve a crucial role in the occurrence and development of osteoporosis $(2,3)$. Runt-related transcription factor 2 (Runx2), a master bone growth regulatory factor, has been thought to be involved in the proliferation and differentiation of osteoblasts $(4,5)$. Studies have reported that physiologic levels of Runx 2 may promote osteoblast function, while abnormal expression of Runx 2 cause the occurrence of bone related diseases $(6,7)$. Puerarin is an important isoflavonoid phytoestrogen extracted from the root of Radix Puerariae, which is widely prescribed for patients in China. Studies demonstrated that puerarin could promote the proliferation, differentiation and mineralization of osteoblasts in vitro $(8,9)$. Previous studies of the authors have also suggested that puerarin may promote the bone formation in MC3T3-E1 osteoblast-like cells $(10,11)$. In a recent report, puerarin was demonstrated to ameliorate bone loss in estrogen-deficient rats, which indicated that puerarin had good anti-osteoporotic effect and may prevent osteoporosis for postmenopausal women (12). Although experiments have indicated that puerarin served an important role in osteoblasts and osteoporosis in vivo and in vitro, the exact anti-osteoporotic mechanism of puerarin remains unclear.

MicroRNAs (miRs) are small single-stranded non-coding RNAs, 18-22 nucleotides in length, which have emerged as a class of important gene regulators on cellular functions in recent years (13). They are known to suppress protein expression by binding to the 3'-untranslated region (UTR) of their target mRNAs (14). It has been suggested that miRNAs may be involved in regulating cell proliferation, differentiation and apoptosis by targeting their downstream genes $(15,16)$. However, specific miRNAs in the regulation of osteoblasts activities have not been well characterized and the mechanisms by which these miRNAs target gene expression in osteoblasts remain elucidated.

In the present study, the promoting effects of puerarin on osteoblasts viability and differentiation were examined and the authors indicated that the expression of Runx 2 was significantly increased in osteoblasts following puerarin treatment, while that of miR-204 was downregulated. Following this, the 
authors explored the underlying target relationship between miR-204 and Runx2 using a dual luciferase reporter gene assay. Furthermore, by modulating miR-204 activity, overexpression of miR-204 was markedly decreased the protein level of Runx2, whereas miR-204 inhibition reversed the effect, indicating that Runx 2 was a direct target gene of miR-204. Together, these findings suggested that puerarin promotes the viability and differentiation of MC3T3-E1 cells by increasing the expression of Runx2 via miR-204 downregulation.

\section{Materials and methods}

Osteoblast culture. MC3T3-E1 cells were purchased from the Shanghai Cell Bank of Type Culture Collection of Chinese Academy of Sciences (Shanghai, China). Cells were cultured in $25 \mathrm{~cm}^{2}$ flasks in $\alpha$-modified Eagle's medium ( $\alpha$-MEM; Wisent Inc., St. Bruno, QC, Canada) supplemented with $10 \%$ fetal bovine serum (FBS, Gibco; Thermo Fisher Scientific, Inc., Waltham, MA, USA), $100 \mathrm{U} / \mathrm{ml}$ penicillin and $100 \mu \mathrm{g} / \mathrm{ml}$ streptomycin, maintained at $37^{\circ} \mathrm{C}$ in a humidified $5 \% \mathrm{CO}_{2}$ incubator (Sanyo Electric Co., Ltd., Moriguchi, Japan).

MTT assay on osteoblasts viability. Briefly, $5 \times 10^{3}$ cells/well were seeded in 96-well culture plates (Corning Incorporated, Corning, NY, USA). The cell culture medium was discarded after $24 \mathrm{~h}$, and $180 \mu \mathrm{l}$ different final concentrations $(0.01,0.1$ and $1 \mathrm{mg} / \mathrm{ml}$ ) puerarin were added to the experimental wells, while an equal volume of serum-free medium was added to the control wells. Following culturing for 24,48 and $72 \mathrm{~h}$, the proliferation of osteoblasts was measured by adding $20 \mu \mathrm{l}$ MTT (5 mg/ml, Amresco LLC, Solon, OH, USA) in each well and incubating for another $4 \mathrm{~h}$. A total of $150 \mu \mathrm{l}$ dimethyl sulfoxide was added in each well. Following this, the supernatant was removed and absorbance was measured at $490 \mathrm{~nm}$ after oscillation for 10 min using a microplate reader (BioTek Instruments, Inc., Winooski, VT, USA). The viability of osteoblasts after transfection with miR-204 mimics, inhibitor or miR negative control was also examined using MTT assay.

Alkaline phosphatase (ALP) activity expression of osteoblasts. Osteoblasts were cultured in a 24 -well culture plate. Following treatment with different final concentrations $(0.01$, 0.1 and $1 \mathrm{mg} / \mathrm{ml}$ ) puerarin for $48 \mathrm{~h}$, cells were washed three times with PBS. The ALP positive cells were stained and ALP activity was assayed using a commercial kit (Nanjing Jiancheng Bioengineering Institute, Nanjing, China) according to the manufacturer's instructions. The ALP activities were also examined in osteoblasts following transfection with miR-204 mimics, inhibitor or miR negative control.

Reverse transcription-quantitative polymerase chain reaction (RT-qPCR). Osteoblasts were seeded in a 24-well plate in $\alpha$-MEM (Wisent, Inc.), supplemented with 10\% FBS (Gibco; Thermo Fisher Scientific, Inc.), at the density of $1 \times 10^{5}$ cells/well. The concentration of $0.01,0.1$ and $1 \mathrm{mg} / \mathrm{ml}$ puerarin was added to the experimental wells, and cultured in a humidified $5 \%$ $\mathrm{CO}_{2}$ incubator at $37^{\circ} \mathrm{C}$ for $48 \mathrm{~h}$. Total RNA was prepared using TRIzol reagent (Invitrogen; Thermo Fisher Scientific, Inc.). Purity and concentration of the isolated RNA were detected by a nucleic acid protein analyzer (BioDrop $\mu$ Lite; Biodrop,
Cambridge, UK). The RNA was then reversed-transcribed to cDNA with a reverse transcription kit (Takara Bio, Inc., Otsu, Japan), following the manufacturer's protocol. The final reaction solution $(25 \mu \mathrm{l})$ contained $2 \mu \mathrm{l}$ cDNA product, $12.5 \mu \mathrm{l} 2 \mathrm{X}$ Realtime PCR SYBR premix (Takara Bio, Inc.), $0.5 \mu 1$ each of forward and reverse primers and $9.5 \mu \mathrm{l}$ distilled water. The two-step PCR amplification conditions were: Pre-denaturation of $95^{\circ} \mathrm{C}$ for $30 \mathrm{sec}$, followed by 40 cycles of $95^{\circ} \mathrm{C}$ for $5 \mathrm{sec}$, $60^{\circ} \mathrm{C}$ for $34 \mathrm{sec}$. The fluorescence signal detection was measured by Mx3000P real-time PCR system (Stratagene; Agilent Technologies, Inc., Santa Clara, CA, USA). Target genes included transforming growth factor (TGF)- $\beta 1$, Smad2, Smad 3 and Runx2, while $\beta$-actin served as an internal control. Primers were designed and synthesized by GenScript (Piscataway NJ, USA) and are presented in Table I.

Western blot analysis. Protein was extracted from the cells with total protein extraction kit (cat. no. BC3710; Beijing Solarbio Science \& Technology Co., Ltd., Beijing, China) after treatment with $0.01,0.1$ and $1 \mathrm{mg} / \mathrm{ml}$ puerarin for $48 \mathrm{~h}$, and then the protein content was measured by the bicinchoninic acid assay method, according to the manufacturer's instructions (cat. no. PC0020; Beijing Solarbio Science \& Technology Co., Ltd.). For western blot analysis, $25 \mu \mathrm{g}$ of samples were subjected to SDS-PAGE gel and electro-transferred onto a polyvinylidene difluoride membrane. The membrane was then blocked with $5 \%$ skim milk in TBS containing $0.1 \%$ Tween-20 (TBST) for $1 \mathrm{~h}$ at room temperature. Following washing three times in TBST for $5 \mathrm{~min}$, the protein samples were incubated with primary antibodies (Cell Signaling Technology, Inc., Danvers, MA, USA) for TGF- $\beta 1$ (cat. no. 709), Smad2 (cat. no. 3122), Smad3 (cat. no. 9523), Runx2 (cat. no. 12556) and $\beta$-actin (cat. no. 4970; all 1:1,000) at $4^{\circ} \mathrm{C}$ overnight. Following washing another three times with TBST, the samples were incubated with horseradish peroxidase-conjugated anti-rabbit IgG secondary antibodies (cat. no. 7074; 1:3,000; Cell Signaling Technology, Inc.) for $1 \mathrm{~h}$ at room temperature. Following a final wash of TBST, the protein bands of interest were visualized by the enhanced chemiluminescence detection system (Bio-Rad Laboratories, Inc., Hercules, CA, USA). The intensity of the bands was measured using the ImageJ2x v2.1.4.7 software (National Institutes of Health, Bethesda, MD, USA) to assess the relative protein levels.

Expression profile analysis of miRNAs. Expression profiles analysis of miRNAs was performed by KangChen Biology Engineering Co., Ltd. (Shanghai, China) following treatment of osteoblasts with $0.1 \mathrm{mg} / \mathrm{ml}$ puerarin for $48 \mathrm{~h}$.

Network prediction for Runx2-targeting miRNAs. The Runx2-targeting miRNAs were predicted using TargetScan (http://genes.mit.edu/targetscan), PicTar (http://pictar.bio.nyu .edu), miRBase (http://mirbase.org/) and miRDB (http://mirdb .org/miRDB/) programs, and then compared with the results of expression profile analysis to determine the Runx2-targeting miRNAs.

Validation of Runx2-targeting miRNA by $R T$ - $q P C R$. Following incubating osteoblasts with puerarin $(0.1 \mathrm{mg} / \mathrm{ml})$ for $48 \mathrm{~h}$, total RNA was extracted using TRIzol reagent (Takara Bio, Inc.). 
Table I. Reverse transcription-quantitative polymerase chain reaction.

\begin{tabular}{lll}
\hline Gene & \multicolumn{1}{c}{ Forward sequence $\left(5^{\prime}-3^{\prime}\right)$} & \multicolumn{1}{c}{ Reverse sequence $\left(5^{\prime}-3^{\prime}\right)$} \\
\hline TGF- $\beta 1$ & CTCCCGTGGCTTCTAGTGC & GCCTTAGTTTGGACAGGATCTG \\
Smad2 & ATGTCGTCCATCTTGCCATTC & AACCGTCCTGTTTTCTTTAGCTT \\
Smad3 & CACGCAGAACGTGAACACC & GGCAGTAGATAACGTGAGGGA \\
Runx2 & CGGACGAGGCAAGAGTTTCA & GGATGAGGAATGCGCCCTAA \\
-actin & GTGCTATGTTGCTCTAGACTTCG & ATGCCACAGGATTCCATACC
\end{tabular}

TGF- $\beta 1$, transforming growth factor- $\beta 1$; Runx 2 , Runt-related transcription factor 2 .

The reverse-transcription reaction solution $(20 \mu \mathrm{l})$ contained $10.0 \mu 1$ 5X Reverse Transcription Mix (Takara Bio, Inc.), $1.0 \mu \mathrm{l}$ Stem-loop RT primers (GenScript), 500 ng miRNA, $2.0 \mu \mathrm{l}$ HiScript Enzyme Mix (cat. no. R223; Vazyme, Piscataway, NJ, USA) and RNase-free water. The PCR amplification conditions were: $25^{\circ} \mathrm{C}$ for $5 \mathrm{~min}, 45^{\circ} \mathrm{C}$ for $50 \mathrm{~min}, 85^{\circ} \mathrm{C}$ for $50 \mathrm{~min}$. Specific miRNA stem-loop RT primers for mouse miR-204 and internal control U6 were designed and synthesized by Shanghai Sangon Pharmaceutical Co. Ltd. (Shanghai, China). Primers used in the stem-loop RT-qPCR are as following: RT primer, 5'-GTCGTATCCAGTGCAGGGTCCGAGGTATTC GCACTGGATACGACAGGCAT-3'; PCR upstream primer, 5'-GCGGCGGTTCCC TTTGTCATCC-3'; downstream primer 5'-ATCCAGTGCAGGGTCCGAGG-3' for miR-204; PCR upstream primer, 5'-CTCGCTTCGGCAGCACA-3'; downstream primer, 5'-AACGCTTCACGAATTTG CGT-3' for U6.

Construction of recombinant plasmid. Empty vectors were prepared for constructing Runx 2 expression vectors, sequences of the wild-type Runx2 3'-UTR (ttctatgcacgtattgtacaaattgtget ttgtgccacaggtcatgatcgtggatgagtttactctgaacttcaaagggactatttgtatt gtatgttgcaactgtaaattgaattatttggcatttccccetctcatgattgtaatatt) and mutant Runx2 3'-UTR (ttctatgcacgtattgtacaaattgtgctttgtgec acaggtcatgatcgtggatgagtttactctgaacttcaaatccactatttgtattgtatgttg caactgtaaattgaattatttggcatttcccctctcatgattgtaatatt) were inserted into the empty vectors. The recombinant plasmids were synthesized by Shanghai GenePharma Co, Ltd. (Shanghai, China).

Dual luciferase reporter gene assay. Osteoblasts were seeded in 24 -well plates at a density of $2 \times 10^{4}$ per well. At $24 \mathrm{~h}$ incubation, cells in each well were transfected with the recombinations of wildtype Runx2 3'-UTR or mutant Runx2 3'-UTR plasmids (100 ng) and miR-204 mimics $(2 \mu \mathrm{M})$ or miR control $(2 \mu \mathrm{M})$ using Lipofectamine 2000 (1 ml; Invitrogen; Thermo Fisher Scientific, Inc.). Cells were collected following $48 \mathrm{~h}$ transfection, then firefly and Renilla luciferase activities were analyzed using Dual-Luciferase Reporter Assay System (Promega Corporation, Madison, WI, USA). The ratio of firefly to Renilla luciferase activity was used as relative luciferase activity.

Overexpression and inhibition of $m i R-204$. The mouse miR-204 mimics, miR-204 inhibitor and negative control were obtained from Shanghai GenePharma Co., Ltd. Cells were seeded in six-well plates at a density of $1 \times 10^{5}$ per well and transfected with $16 \mu$ l RNAs using $4 \mu$ l Lipofectamine 2000 transfection reagent (Invitrogen; Thermo Fisher Scientific, Inc.) when the cells reached $90 \%$ confluence. Control cells were only transfected with $4 \mu 1$ transfection reagent. Then cells were cultured in a humidified $5 \% \mathrm{CO}_{2}$ incubator at $37^{\circ} \mathrm{C}$ for $48 \mathrm{~h}$. Cells were ready for RT-qPCR and western blot analysis of Runx2. Viability and ALP activity were also examined.

Statistical analysis. The results were presented as mean \pm standard deviation. Data comparisons were analyzed by Student's t-test or one-way analysis of variance with SPSS software (version, 17.0; SPSS Inc., Chicago, IL, USA). Comparisons between groups were analyzed using the Bonferroni post hoc test. $\mathrm{P}<0.05$ was considered to indicate a statistically significant difference.

\section{Results}

Puerarin promotes osteoblast viability and differentiation. The authors first examined whether puerarin could affect osteoblast viability. Osteoblasts were cultured with puerarin at various concentrations. The cells treated with puerarin had significantly higher viability compared with that of the control cells $(\mathrm{P}<0.05)$, except those treated with puerarin at a lower concentration $(0.01 \mathrm{mg} / \mathrm{ml})$ for $24 \mathrm{~h}$ and $48 \mathrm{~h}$ (Fig. 1A). The osteoblasts treated with puerarin for $48 \mathrm{~h}$ at various concentrations $(0.01,0.1$ and $1 \mathrm{mg} / \mathrm{ml})$ had significantly higher ALP activity than that of the control cells $(\mathrm{P}<0.05$; Fig. 1B). These data suggested that puerarin may promote osteoblast viability and differentiation.

Puerarin increases the expression of TGF- $\beta 1$, Smad2, Smad3 and Runx2. In order to explore the proliferation effect of puerarin on osteoblasts, the authors studied the expression of osteoblast-related regulatory factors (TGF- $\beta 1$, Smad2, Smad3 and Runx2). Following treating cells with puerarin for $48 \mathrm{~h}$, we measured TGF- $\beta 1$, Smad 2, Smad 3 and Runx 2 gene expression with RT-qPCR and also the protein expression with western blotting. The transcription levels and the translation levels of the genes were all significantly increased in the cells treated with puerarin $(\mathrm{P}<0.05$; Fig. 2). The data imply that these factors may be involved in the promotion effects of puerarin on osteoblasts. Studies have indicated that Runx2 is a master regulatory factor that serves a crucial role on the regulation of 

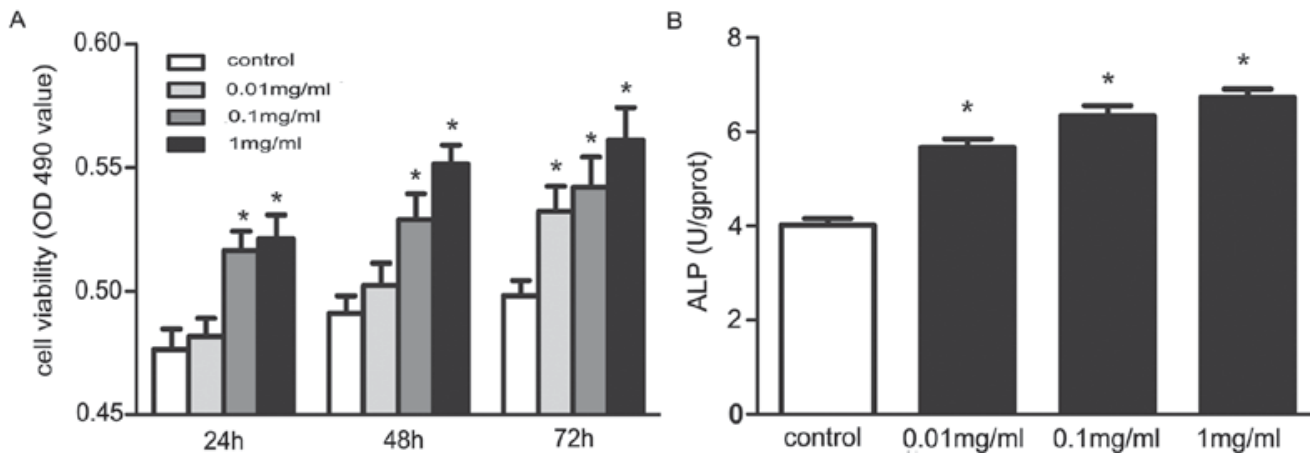

Figure 1. Puerarin promotes the viability and differentiation of osteoblasts. (A) Effect of $0.01,0.1 \mathrm{and} 1 \mathrm{mg} / \mathrm{ml}$ puerarin on osteoblasts by MTT assay, compared with a control with serum-free medium. (B) Effect of $0.01,0.1 \mathrm{and} 1 \mathrm{mg} / \mathrm{ml}$ puerarin on ALP activity of osteoblasts for $48 \mathrm{~h}$. "P $<0.05 \mathrm{vs}$. control. ALP, alkaline phosphatase.

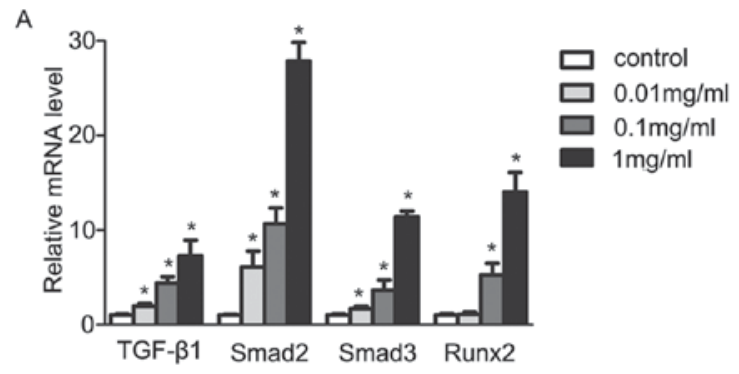

B

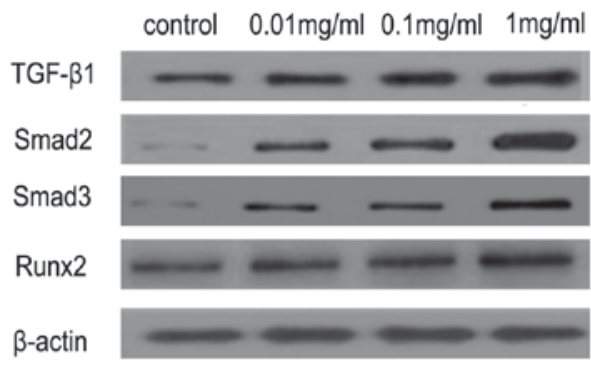

C

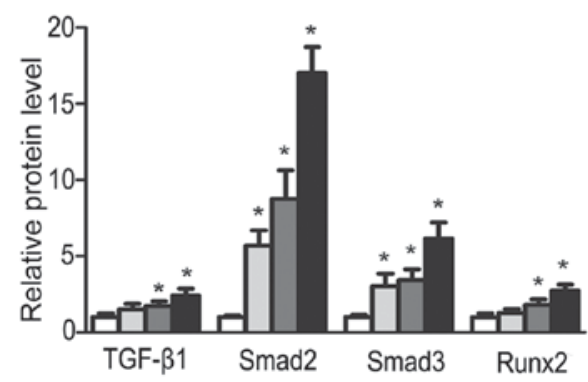

Figure 2. Puerarin promotes the expression of TGF- $\beta 1, \mathrm{Smad} 2, \mathrm{Smad} 3$ and Runx 2 in osteoblasts. Cells were treated with puerarin $(0.01,0.1,1 \mathrm{mg} / \mathrm{ml})$ for $48 \mathrm{~h}$ before measurement. (A) TGF- $\beta 1, \operatorname{Smad} 2, \operatorname{Smad} 3$ and Runx2 gene transcription levels were assessed by reverse transcription-quantitative polymerase chain reaction. (B) The protein expression levels were measured by western blotting. (C) Quantification of (B). ${ }^{*} \mathrm{P}<0.05$ vs. the control ( $\beta$-actin served as the internal control). TGF- $\beta 1$, transforming growth factor- $\beta 1$; Runx2, Runt-related transcription factor 2.

transcription in osteoblasts. Afterwards, the authors focused on the expression of Runx2-targeting miRNAs (17).

Puerarin downregulates the expression of $m i R-204$. Analysis of miRNA expression profiles demonstrated that the expression of miR-204 and other 118 miRNAs changed following puerarin treatment for $48 \mathrm{~h}$ (18). In addition, miRNA target analysis programs (TargetScan, PicTar, miRBase and miRDB) predicted that miR-204 may directly target the osteogenic crucial regulator Runx2. Then, the effect of puerarin on miR-204 expression was examined by stem-loop RT-qPCR. The expression level of miR-204 was significantly decreased following culturing the cells with $0.1 \mathrm{mg} / \mathrm{ml}$ puerarin for $48 \mathrm{~h}$, compared with that of the cells in absence of puerarin $(\mathrm{P}<0.05$; Fig. 3A). The results suggested that puerarin downregulated the expression of miR-204.

Combined with the previous validation that puerarin promoted osteoblast viability and differentiation through upregulation of Runx2, however, the authors demonstrated that miR-204 was downregulated. Moreover, according to the bioinformatics analysis, miR-204 was suggested to bind to the 3'-UTR of Runx2 through specific binding sites (Fig. 3B). Taken together, future studies are prompted to determine whether Runx 2 is a direct downstream target of miR-204.

Runx2 is the target of miR-204 in mouse osteoblasts. To further investigate whether Runx 2 can be directly targeted by miR-204, luciferase reporters containing wildtype or mutant 3'-UTR of Runx2 were engineering and transfected with miR-204 mimics or miR negative control. As presented in Fig. 4A, transfection with miR-204 mimics of wildtype-Runx2-3'-UTR recombinant plasmid had lower luciferase activity compared with that of the miR negative control, while transfection with miR-204 mimics of mutant-Runx2-3'-UTR recombinant plasmid had no statistically significant difference compared with that of the miR negative control. These data 
A

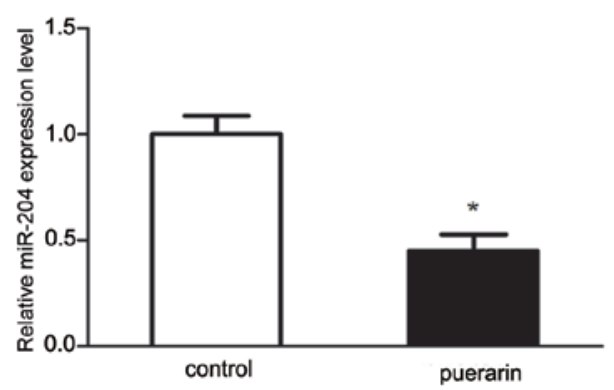

B

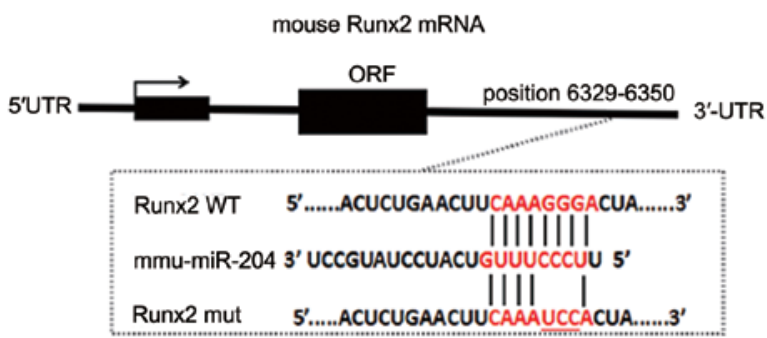

Figure 3. Puerarin downregulates miR-204 expression. (A) The expression levels were measured following cells were treated with $0.1 \mathrm{mg} / \mathrm{ml}$ puerarin for $48 \mathrm{~h}$. ${ }^{*} \mathrm{P}<0.05$ vs. the control. (B) Schematic of the miR-204 target sites within the mouse Runx2 WT and Runx2 mut 3'-UTR, the three mutated nucleotides are underlined. miR, microRNA; Runx2, Runt-related transcription factor 2; UTR, untranslated region; ORF, open reading frame; mut, mutant.

A

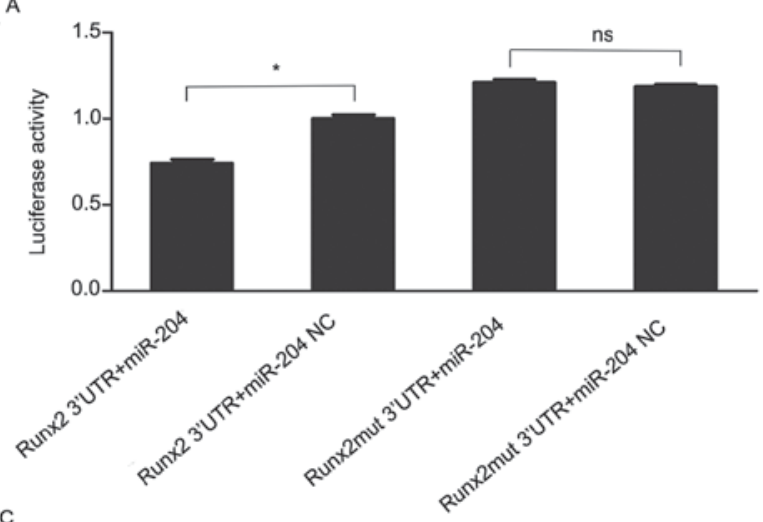

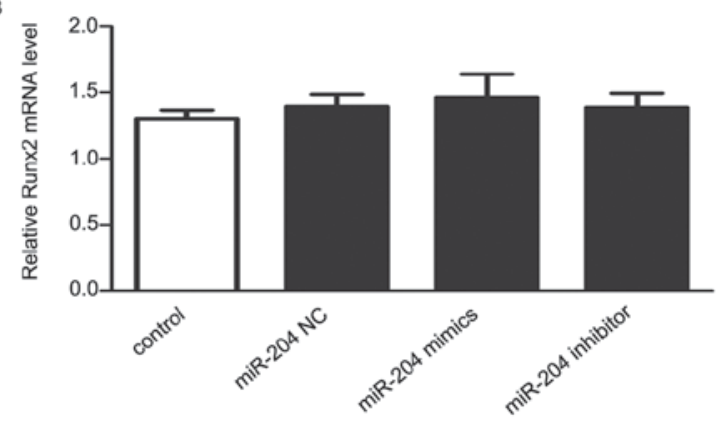

D

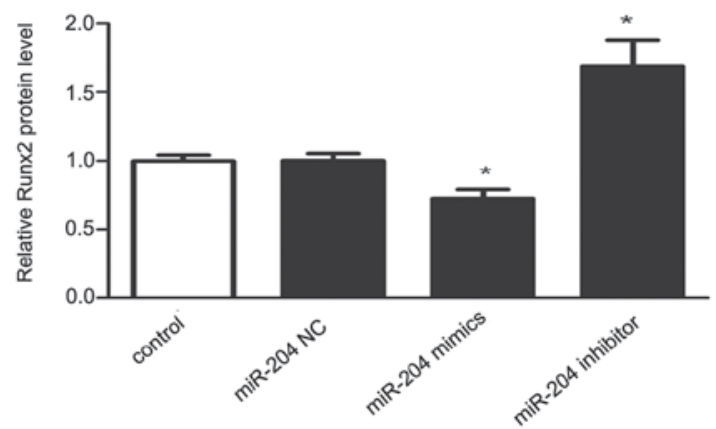

Figure 4. miR-204 directly targets Runx2. (A) Wild type or mutant Runx2-3'-UTR reporter transfected with miR-204 mimics or miR negative control into osteoblast cells. At 48 h, luciferase activities were measured. Relative luciferase activity=(Renilla/Firefly) ratio. (B) Reverse transcription-quantitative polymerase chain reaction and (C and D) western blotting were used to assess the expression of Runx 2 in osteoblasts at $48 \mathrm{~h}$ following transfection with miR-204 mimics, inhibitor or miR negative control. ${ }^{*} \mathrm{P}<0.05$ as indicated. miR, microRNA; Runx2, Runt-related transcription factor 2; UTR, untranslated region; NC, negative control; mut, mutant.

indicated that miR-204 dramatically repressed the luciferase activity of the reporter containing the wild-type full-length 3'-UTR of Runx2, but not the mutant full-length 3'-UTR of Runx2, implying that miR-204 may directly target Runx2.

The effects of overexpression and inhibition of miR-204 on Runx 2 were then investigated. The expression of Runx2 was analyzed using RT-qPCR and western blotting methods in osteoblasts at $48 \mathrm{~h}$ following transfection with miR-204 mimics, inhibitor or miR negative control. The results of RT-qPCR (Fig. 4B) did not show significant difference, but western blotting analysis (Figs. 4C and D) indicated that overexpression of miR-204 in osteoblasts significantly decreased the expression level of Runx2, while inhibition of miR-204 had the opposite effect. The effects of alternation on protein level, rather than on mRNA level, suggesting that a role of miR-204 for post-transcriptional regulation. Collectively, these results indicated that Runx2 is a direct target of miR-204.

miR-204 inhibits the viability and differentiation of osteoblasts. Finally, the authors investigated the effect of miR-204 on osteoblasts. Following transfection with miR-204 mimics, inhibitor or miR negative control for $48 \mathrm{~h}$, the viability and ALP activity of osteoblasts were tested. The cells that were transfected with miR-204 mimics had significantly lower viability, but cells transfected with miR-204 inhibitor had higher viability compared with that of the control cells (Fig. 5A). Similarly, transfection with miR-204 mimics significantly reduced ALP activity, whereas transfection with 
A

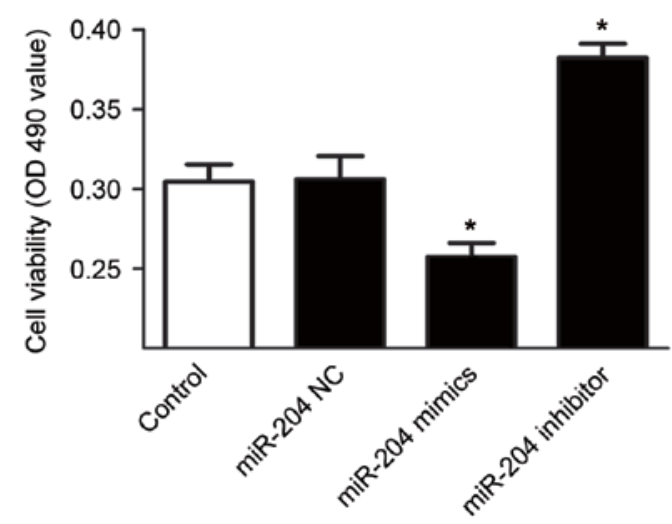

B

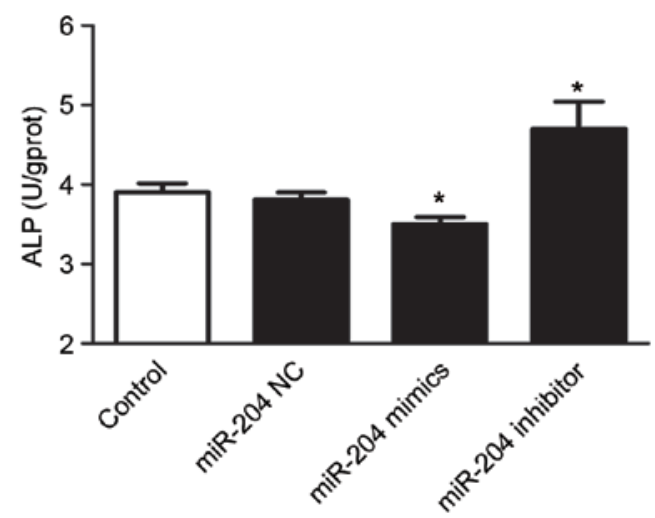

Figure 5. miR-204 inhibits osteoblast viability and differentiation. (A) Following transfecting cells with miR-204 mimics, inhibitor or miR negative control for $48 \mathrm{~h}$, the viability was tested by MTT assay. (B) ALP activity of osteoblasts for $48 \mathrm{~h}$ was assayed by a commercial kit. * $<0.05 \mathrm{vs}$. the control. miR, microRNA; ALP, alkaline phosphatase; NC, negative control; OD, optical density.

miR-204 inhibitor had the opposite effect (Fig. 5B). These results suggested that miR-204 may inhibit osteoblast viability and differentiation.

\section{Discussion}

Runx 2 is an important bone regulatory factor, which serves a crucial role in the regulation of transcription in osteoblasts. Puerarin was reported to promote the proliferation and osteoblastic differentiation in human bone marrow stromal cells by increasing the expression of osteoblastic markers, including Runx2 (19). Runx2, BMP-2 and OPG gene expression was found to be upregulated following treating mouse osteoblasts $1 \times 10^{-8} \mathrm{~mol} / 1$ puerarin (20). However, other research reported that puerarin significantly increased the expression of ALP, OPG and RANKL, but not Runx2 in UMR106 cells (8). In addition, puerarin increased the mRNA levels of osteoblast differentiation markers, such as ALP and type I collagen, but not Runx 2 or osterixin in primary baboon osteoblasts (21). Obviously, the effects of puerarin on Runx 2 in different cells remains a controversial issue. In the current study, puerarin significantly increased the expression of Runx 2 in osteoblast-like MC3T3-E1 cells, and the further study demonstrated that miR-204 was involved in the regulatory mechanism.

miRNAs are a large family of small non-coding RNAs that regulate gene expression. miR-204, a negative regulator, is involved in the regulation of many biological activities. It is validated that the expression of miR-204 is downregulated in bone tissue of ovariectomized mice (22) and it is also reported miR-204 has no effect on bone formation in vivo (23). However, miR-204 has received little attention in osteoblast viability and differentiation in vitro. In the present study, puerarin promoted the viability and differentiation of osteoblasts, and correspondingly increased the expression of Runx 2 and downregulated the expression level of miR-204. The results indicated that miR-204 and Runx 2 may be involved in the regulation of the osteoblast viability and differentiation, and then the authors sought to clarify the relationship between miR-204 and Runx2. In the present study, the bioinformatics analysis demonstrated that miR-204 could bind to the 3'-UTR of Runx 2 through specific binding sites. miR-204 dramatically reduced the luciferase activity of wild type Runx2 3'-UTR transfected cells, but not that of the mutant ones. Moreover, overexpression of miR-204 significantly decreased the protein expression level of Runx2, while inhibition of miR-204 enhanced the level. The effect of alternation on protein levels, rather than mRNA level, suggesting a role of miR-204 for post-transcriptional regulation. Taken together, the current study demonstrated that Runx2 is a direct target of miR-204. Consistently, Huang et al (24) demonstrated that miR-204, functioning as an endogenous negative regulator of Runx2, inhibited osteogenesis and promotes adipogenesis of mesenchymal progenitor cells and bone marrow mesenchymal stem cells (24). Wang et al (25) also confirmed that miR-204 inhibited osteogenesis of human aortic valve interstitial cells by negatively regulating the expression of Runx 2 .

In the current study, the authors observed and explored the change of Runx 2 targeted by miR-204; however, some other osteoblast-related regulatory factors such as TGF- $\beta 1, \mathrm{Smad} 2$ and Smad3 were upregulated following treatment of puerarin. Although miR-204 could not directly target these factors based on software prediction and bioinformatics analysis, it has been revealed that miRNAs usually function in clusters (26). The mechanism whether the other varied miRNAs among the miRNA expression profiles after puerarin treatment has effects on TGF- $\beta 1$, Smad 2 and Smad 3 remains unknown. In fact, studies have indicated that Runx2, TGF- $\beta 1$, Smad 2 and Smad3 are capable of regulating the expression of miRNAs directly or indirectly (27-29), suggesting that regulatory factors and miRNAs are not independent and they regulate each other bidirectionally. In view of the promoting effect of puerarin on cellular viability and differentiation, its regulatory effects on miR-204 and the downstream target Runx2, the detailed upstream signaling pathway by which puerarin affects miR-204 downregulation is still unknown, which remains to be elucidated. Besides, further research on human osteoblasts is required to further confirm these findings because of the differences between mouse and human cells.

In conclusion, the authors demonstrated that puerarin significantly promoted the viability and differentiation of osteoblasts. Following treatment with puerarin in mouse osteoblasts, the expression level of Runx 2 was significantly 
increased, which was negatively correlated with that of miR-204, whose expression level was decreased. Moreover, the current study demonstrated that miR-204 could directly target Runx2; Runx2 was identified as a target of miR-204. The results suggested that puerarin promoted the viability and differentiation of mouse osteoblasts by increasing the expression of Runx2 via miR-204 downregulation. These findings provide a better understanding of the biological effects of puerarin on bone formation in vitro, and suggested the potential use of puerarin in prevention and treatment of osteoporosis.

\section{Acknowledgements}

The present study was supported by the Natural Science Foundation of Jiangsu Province (grant no. BK20131417) and by A Project Funded by the Priority Academic Program Development of Jiangsu Higher Education Institutions (Integration of Chinese and Western Medicine).

\section{References}

1. NIH Consensus Development Panel on Osteoporosis Prevention, Diagnosis, and Therapy: Osteoporosis prevention, diagnosis, and therapy. JAMA 285: 785-795, 2001.

2. Rachner TD, Khosla S and Hof bauer LC: Osteoporosis: Now and the future. Lancet 377: 1276-1287, 2011.

3. An J, Yang H, Zhang Q, Liu C, Zhao J, Zhang L and Chen B: Natural products for treatment of osteoporosis: The effects and mechanisms on promoting osteoblast- mediated bone formation. Life Sci 147: 46-58, 2016

4. Haxaire C, Haÿ E and Geoffroy V: Runx2 controls bone resorption through the down-regulation of the Wnt pathway in osteoblasts. Am J Pathol 186: 1598-1609, 2016.

5. Wysokinski D, Pawlowska E and Blasiak J: RUNX2: A master bone growth regulator that may be involved in the DNA damage response. DNA Cell Biol 34: 305-315, 2015.

6. Sun C, Qiu Y, Yin G, Shu H, Liu Z, Wang XH, Liu WJ and Li HB: Abnormal expression and significance of Runx2 in osteoblasts of adolescent idiopathic scoliosis patients. Zhonghua Wai Ke Za Zhi 47: 1495-1498, 2009 (In Chinese).

7. Li N, Luo D, Hu X, Luo W, Lei G, Wang Q, Zhu T, Gu J, Lu Y and Zheng Q: RUNX2 and osteosarcoma. Anticancer Agents Med Chem 15: 881-887, 2015.

8. Tiyasatkulkovit W, Charoenphandhu N, Wongdee K, Thongbunchoo J, Krishnamra $\mathrm{N}$ and Malaivijitnond S: Upregulation of osteoblastic differentiation marker mRNA expression in osteoblast-like UMR106 cells by puerarin and phytoestrogens from Pueraria mirifica. Phytomedicine 19: $1147-1155,2012$.

9. Wang C, Meng MX, Tang XL, Chen KM, Zhang L, Liu WN and Zhao YY: The proliferation, differentiation, and mineralization effects of puerarin on osteoblasts in vitro. Chin J Nat Med 12: 436-442, 2014

10. Zhan XQ, Qian KQ and Sun YM: Action of puerarin on TGF- $\beta 1 /$ Smad pathway in MC3T3-E1 cells. Chin Tradition Patent Med 35: 1121-1124, 2013 (In Chinese).

11. Dong BS, Zhan XQ and Sun YM: Effects of Puerarin on OPG/RANKL system with culture of osteoblast in vitro. Jilin J Tratitional Chin Med 32: 388-390, 2012 (In Chinese).
12. Suthon S, Jaroenporn S, Charoenphandhu N, Suntornsaratoon $P$ and Malaivijitnond S: Anti-osteoporotic effects of Pueraria candollei var. mirifica on bone mineral density and histomorphometry in estrogen-deficient rats. J Nat Med 70: 225-233, 2016.

13. Bartel DP: MicroRNAs: Genomics, biogenesis, mechanism, and function. Cell 116: 281-297, 2004.

14. Yates LA, Norbury CJ and Gilbert RJ: The long and short of microRNA. Cell 153: 516-519, 2013.

15. Wei J, Shi Y, Zheng L, Zhou B, Inose H, Wang J, Guo XE, Grosschedl R and Karsenty G: miR-34s inhibit osteoblast proliferation and differentiation in the mouse by targeting SATB2. J Cell Biol 197: 509-521, 2012.

16. Chen X, Huang Z, Chen D, Yang T and Liu G: Role of microRNA-27a in myoblast Differentiation. Cell Biol Int 38: 266-271, 2014.

17. Bruderer M, Richards RG, Alini M and Stoddart MJ: Role and regulation of RUNX2 in osteogenesis. Eur Cell Mater 28: 269-286, 2014

18. Zhang YY, Zhou JB, Zeng XW, Zhao FM, Liu GD and Zhan XQ: Effects of puerarin on proliferation of osteoblast and Runx2-targeting miRNAs. Chin Pharmacol Bull 32: 1457-1462, 2016 (In Chinese).

19. Lv H, Che T, Tang X, Liu L and Cheng J: Puerarin enhances proliferation and osteoblastic differentiation of human bone marrow stromal cells via a nitric oxide/cyclic guanosine monophosphate signaling pathway. Mol Med Rep 12: 2283-2290, 2015.

20. Sheu SY, Tsai CC, Sun JS, Chen MH, Liu MH and Sun MG: Stimulatory effect of puerarin on bone formation through co-activation of nitric oxide and bone morphogenetic protein-2/mitogen-activated protein kinases pathways in mice. Chin Med J (Engl) 125: 3646-3653, 2012.

21. Tiyasatkulkovit W, Malaivijitnond S, Charoenphandhu N, Havill LM, Ford AL and VandeBerg JL: Pueraria mirifica extract and puerarin enhance proliferation and expression of alkaline phosphatase and type I collagen in primary baboon osteoblasts. Phytomedicine 21: 1498-1503, 2014.

22. An JH, Ohn JH, Song JA, Yang JY, Park H, Choi HJ, Kim SW, Kim SY, Park WY and Shin CS: Changes of microRNA profile and microRNA-mRNA regulatory network in bones of ovariectomized mice. J Bone Miner Res 29: 644-656, 2014.

23. Cui RR, Li SJ, Liu LJ, Yi L, Liang QH, Zhu X, Liu GY, Liu Y, Wu SS, Liao XB, et al: MicroRNA-204 regulates vascular smooth muscle cell calcification in vitro and in vivo. Cardiovasc Res 96: 320-329, 2012.

24. Huang J, Zhao L, Xing L and Chen D: MicroRNA-204 regulates Runx 2 protein expression and mesenchymal progenitor cell differentiation. Stem Cells 28: 357-364, 2010.

25. Wang Y, Chen S, Deng C, Li F, Wang Y, Hu X, Shi F and Dong N: MicroRNA-204 Targets Runx2 to Attenuate BMP-2-induced osteoblast differentiation of human aortic valve interstitial cells. J Cardiovasc Pharmacol 66: 63-71, 2015.

26. Wang FE, Zhang C, Maminishkis A, Dong L, Zhi C, Li R, Zhao J, Majerciak V, Gaur AB, Chen S and Miller SS: MicroRNA-204/211 alters epithelial physiology. FASEB J 24: 1552-1571, 2010.

27. Chen P, Wei D, Xie B, Ni J, Xuan D and Zhang J: Effect and possible mechanism of network between microRNAs and RUNX2, gene on human dental follicle cells. J Cell Biochem 115: 340-348, 2014.

28. Yin X, Tian W, Wang L, Wang J, Zhang S, Cao J and Yang H: Radiation quality-dependence of bystander effect in unirradiated fibroblasts is associated with TGF- $\beta 1-$ Smad 2 pathway and miR-21 in irradiated keratinocytes. Sci Rep 5: 11373, 2015.

29. García R, Nistal JF, Merino D, Price NL, Fernández-Hernando C, Beaumont J, González A, Hurlé MA and Villar AV: p-SMAD2/3 and DICER promote pre-miR-21 processing during pressure overload-associated myocardial remodeling. Biochim Biophys Acta 1852: 1520-1530, 2015. 